\title{
Received Indicator
}

National Cancer Institute

\section{Source}

National Cancer Institute. Received Indicator. NCI Thesaurus. Code $C 95345$.

An indication as to whether the entity was received. 